\title{
Some utilities to help produce Rich Text Files from Stata
}

\author{
Matthew S. Gillman \\ Wolfson Institute of Preventive Medicine \\ Barts and The London School of Medicine and Dentistry \\ Queen Mary University of London \\ Charterhouse Square \\ London \\ EC1M 6BO \\ UK
}

\begin{abstract}
Producing RTF files from Stata can be difficult and somewhat cryptic. Utilities are introduced to simplify this process; one builds up a table row-by-row, another inserts a PNG image file into an RTF document, and the others start and finish the RTF document.
\end{abstract}

Keywords: Rich Text Format, RTF, automation, PNG, tables, fonts

\section{Introduction}

Users of Stata sometimes wish to generate documents in Rich Text Format (RTF). Such documents are not tied to one particular operating system but are platform-independent. They can be opened (and edited) with word-processing software such as Microsoft Word and Libre Office.

One paradigm in which RTF documents may be required is if a statistician has an analysis which they repeat many times with varying parameters and/or data. It would be tedious (not to say error-prone) if, each time the analysis was re-run, the user had to copy the results from Stata and paste them into the document they were working on. It would be far more convenient (and reproducible) if the statistician used a do-file which consistently performed the same analysis and wrote the results to their document automatically.

The RTF specification was developed by Microsoft and the final version (1.9.1) is available for download (Microsoft Corporation 2008). A key resource is the very useful book written by Sean M. Burke (Burke 2003) which summarises the main features of the specification.

Writing RTF documents is somewhat cryptic. A "Hello world" example might be (excluding the line numbers on the left-hand side):

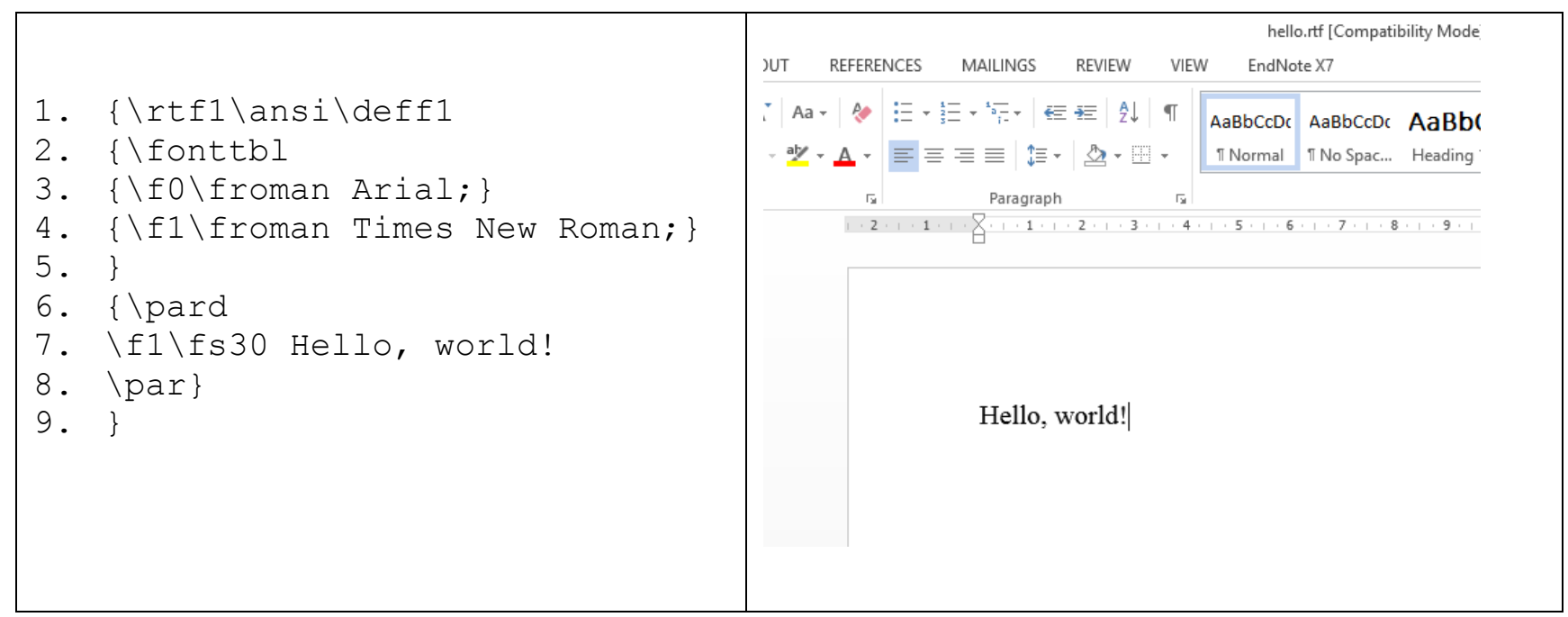


If the content (excluding line numbers) in the left of the preceding table is saved in a text file with the name hello.rtf, it should be viewable in standard word processing packages (e.g. Microsoft Word, Libre Office, etc.) as shown in the right of the table. Note that such a package will display the contents in RTF format; if one wishes to view and debug code like that in the left of the table, one must use an ASCll editor such as Notepad, vi or similar. Here, the first line opens the document and sets the default font to be $f 1$ in the (forthcoming) font table. The second line declares the font table. Lines 3 and 4 define the fonts to be used for fonts $\mathrm{fo}$ and $\mathrm{f1}$ (there can be more than this). In this specific example line 3 is superfluous as font $\mathrm{fO}$ is not used in the document. The closing brace on line 5 ends the font table. The $\{\backslash$ pard command on line 6 opens a new paragraph. Line 7 contains the text for this paragraph, using font $\mathrm{f1}$ from the table (f0 could be used instead, if desired) and in size 15 pt. (Note that RTF requires double this number to be specified as the $f_{S}$ (font size) value, in this case $15 * 2=30$ ). The $\backslash$ par $\}$ on line 8 closes the paragraph. Finally, the closing brace on line 9 completes the RTF document.

In order to insert a table, things get more complicated, especially if one wishes to have visible borders around the table. An ado file has been written to make this a much simpler process (see section 2).

It is also possible to add PNG images into an RTF document, and again a utility has been written to facilitate this (see section 3). Hopefully these utilities will add to those already produced by Roger Newson (author of rtfutil) and others.

Of course, we assume that the RTF document is produced by Stata. Hence the lines above might be placed in a do-file as file write statements, thus:

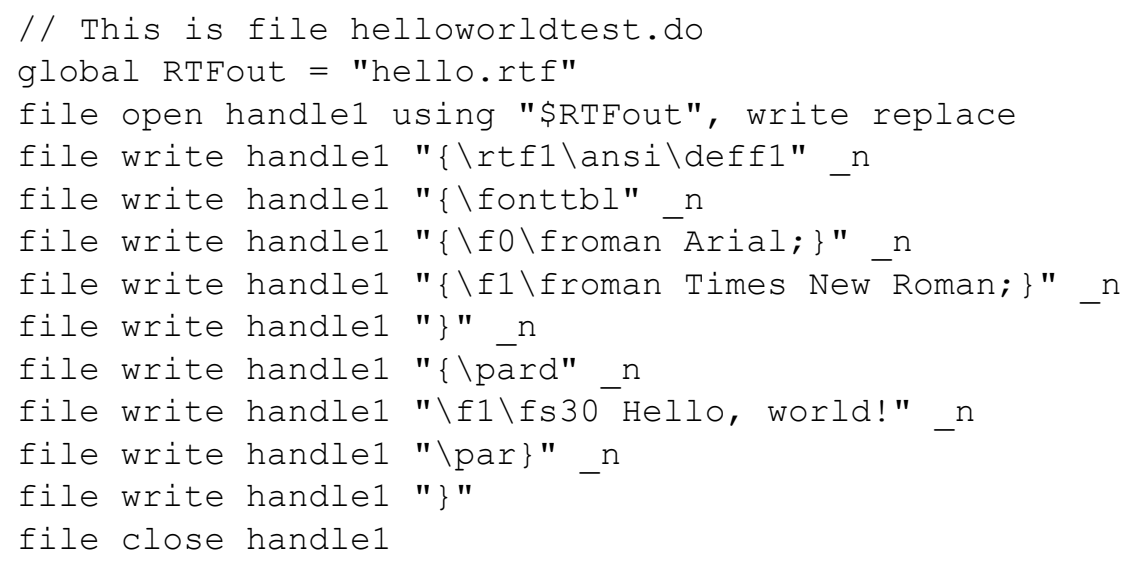

Newlines ( $\mathrm{n}$ ) are not strictly necessary, but aid in the debugging of RTF file syntax.

It is tedious to set up the font table each time one wishes to create an RTF document. Accordingly, a utility called initiatertf has been created, and another (endrtf) to finalise the document. Using these helper functions, the above becomes:

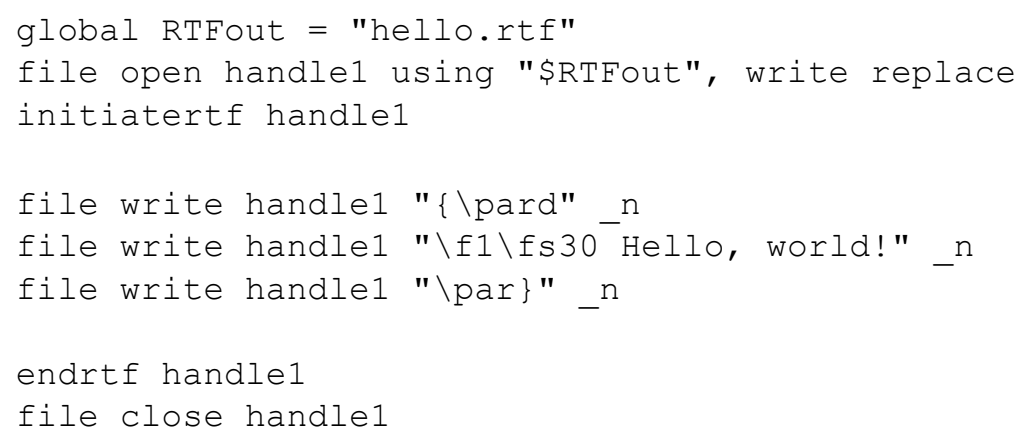


initiatertf has options to specify the fonts used in the document's font table, to set the size of the margins (in $\mathrm{mm}$ ) and also to set the size of the width of the entire page (again in $\mathrm{mm}$ ).

\section{Adding rows to a table}

To write a simple $2 \times 2$ table (without borders) to an RTF file, one might include:

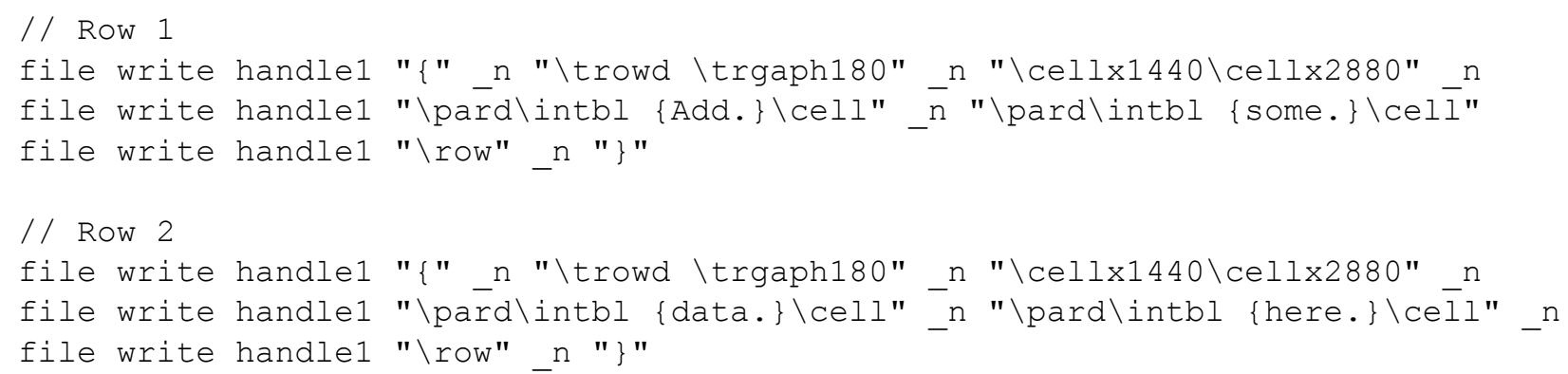

Here is the above code fragment in context, using the helper functions already introduced:

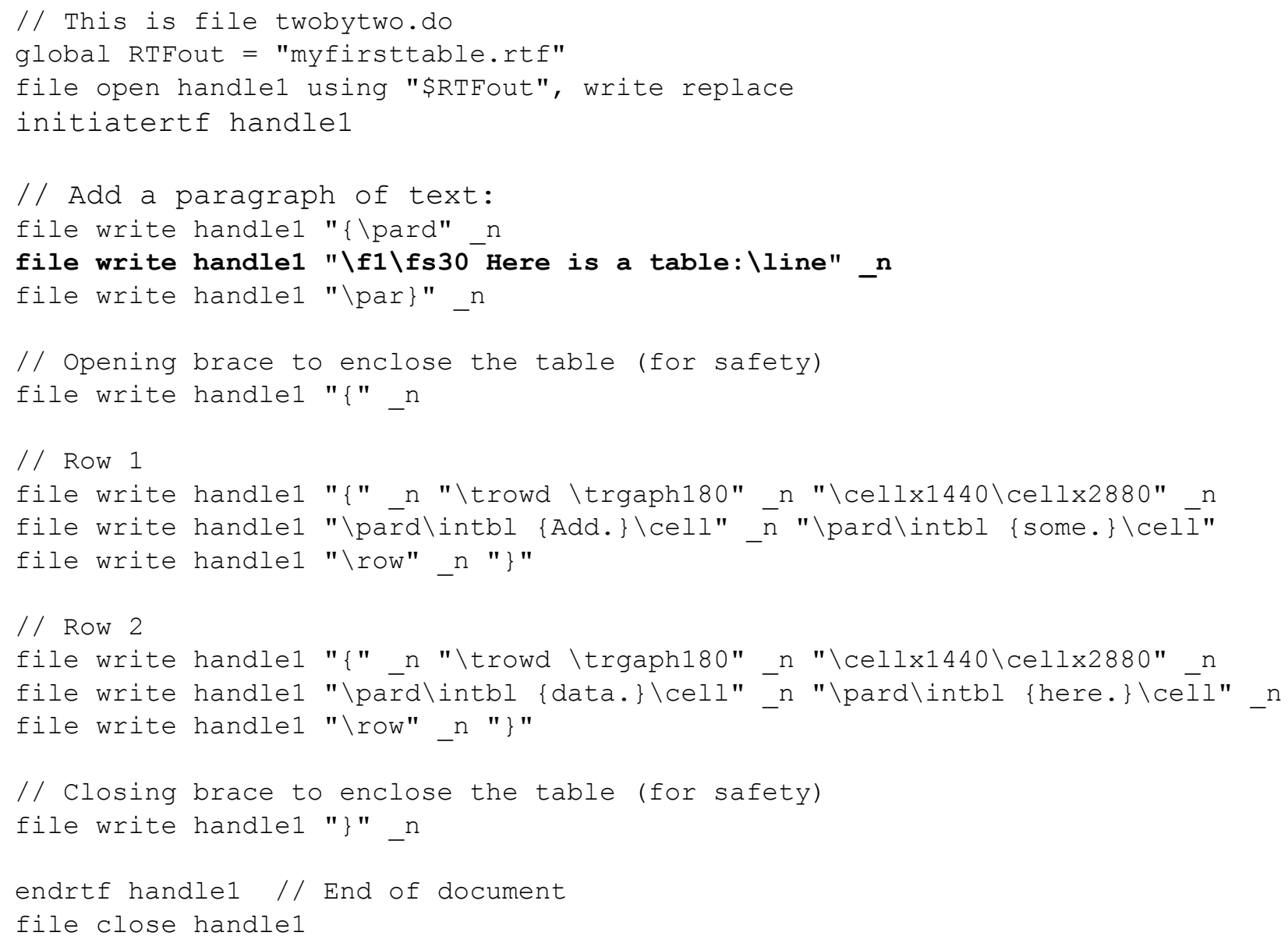

Note that RTF does not require a "table object" to be declared, but builds up a table one row at a time. The Stata above would result in content looking like the following: 


\section{Here is a table:}
Add.
some.
data.
here.

Note that although the Stata newline character $(\mathrm{n})$ can be used to generate a new line in the RTF file generated by Stata, in order to add a blank line within the RTF document as it is displayed by a word processor requires use of $\backslash l$ ine (see bold above). These can be chained together: $\backslash$ line $\backslash$ line $\backslash l$ ine.

Readers will note the increasingly cryptic nature of the RTF text. In this example, based on one in (Burke 2003), the internal margins of the cells are 180 twips $^{1}$. That is what the " $\backslash$ trgaph180" sets up. Starting from the left, the first cell ends at 1,440 twips ( 1 inch) from the margin and the second at 2,880 twips ( 2 inches). Each row is declared to begin with \trowd and end with \row. This example follows the safety recommendation in (Burke 2003) to wrap the text of each cell in braces \{\}, also each table row, and indeed the entire table.

The situation is even more complicated when one wishes to add borders to a table. However, rather than showing the full gory details of this, the first utility is introduced: addrowrtf. It is very simple to use this; a table similar to the above but with borders (the default) would be declared in the do-file as:

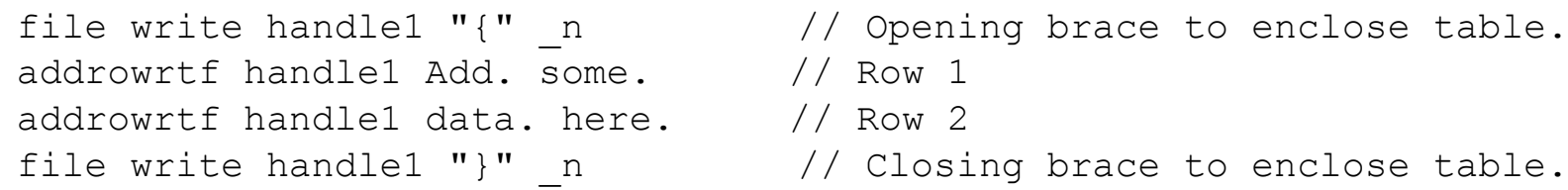

Notice that here the only parameters passed to addrowrtf are the file handle and the text to be entered in the cells. This example will produce a table looking something like this:

\begin{tabular}{|l|l|}
\hline Add. & some. \\
\hline data. & here. \\
\hline
\end{tabular}

The addrowrt $f$ utility has a number of options which can be used. Here, no options were specified, and so the default table font size of 10 point was used. Options are described in the help file, and include the ability to specify the cell width, whether borders are drawn or not, the font size, font number and relative cell widths.

In fact, addrowrtf will dynamically calculate the number of columns, based on the number of arguments supplied. There is no requirement for successive rows to have the same number of cells (columns) as the preceding one(s). An example might be:

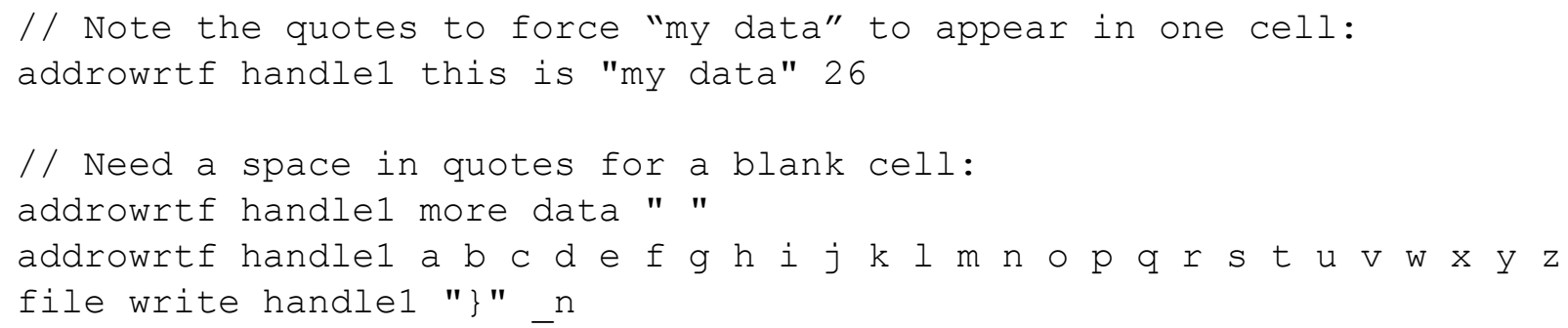

${ }^{1} 1 \mathrm{twip}=1 / 20$ of a typographical point $=1 / 1440$ inch or $\sim 1 / 57 \mathrm{~mm}$. 
which produces:

\begin{tabular}{|l|l|l|l|l|l|l|}
\hline this & is & my data & 26 & \multicolumn{4}{l}{} \\
\hline more & data & & \multicolumn{4}{|c|}{ g } \\
\hline a & b & c & d & e & f & g \\
\hline
\end{tabular}

Here, the rows are positioned using the default offset and cell widths. Using these, the third row expands beyond the margin of the page. Options such as tablewidth and internal may be used to prevent this.

A suitable do-file might look like this:

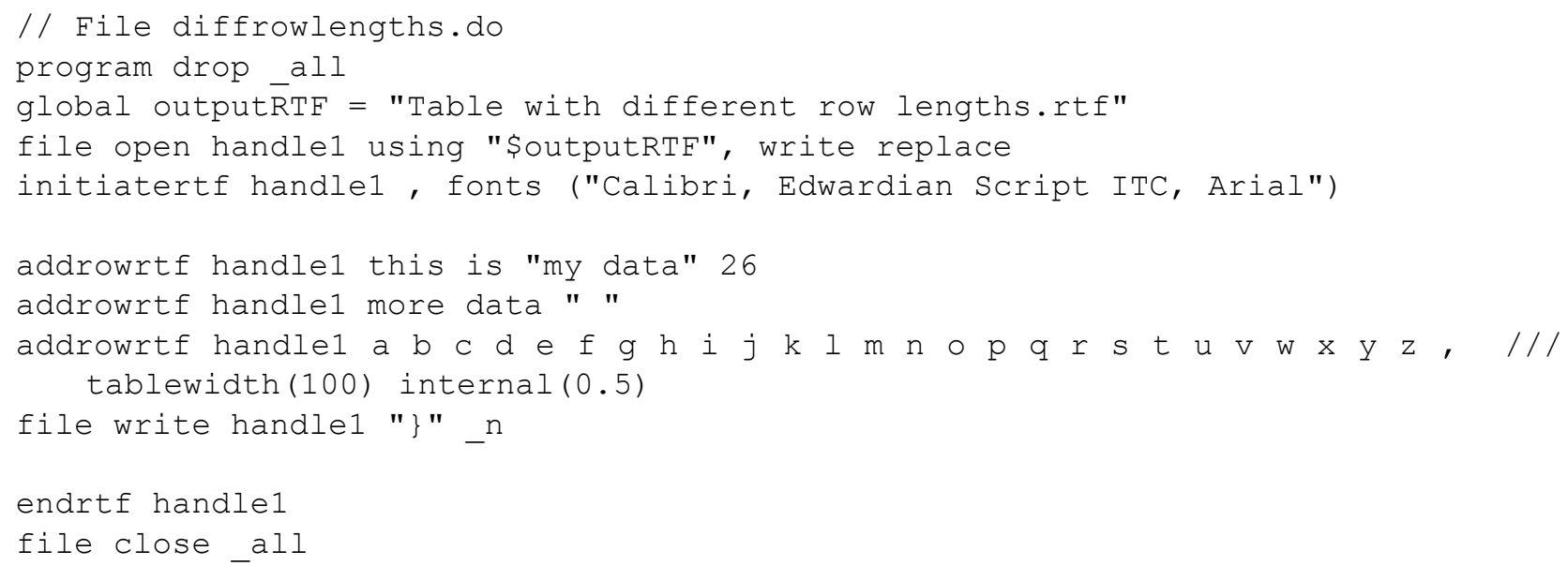

The appearance is thus changed to:

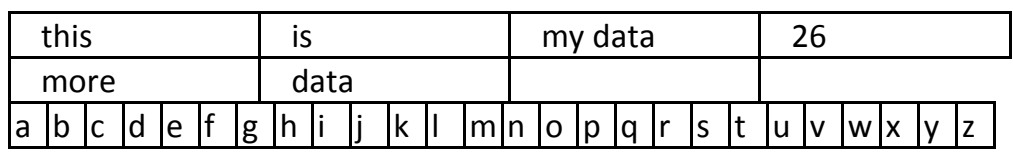

For full details of addrowrtf's options, refer to the help file.

\section{Inserting a PNG image}

It is possible to dynamically create a graph in Stata, save it to a PNG file, and then insert the latter into an RTF document file. It is tricky to do this, not least because Stata must open the PNG file, read a single byte at a time, and convert each byte into hexadecimal format before writing it to the RTF file. Readers will be pleased to know that a utility has been written for just such an eventuality: insertimagertf. Like addrowrtf, it uses a file handle. Austin Nichols has written png2rtf which is similar although it requires a filename to be provided and has more options; insertimagertf is a simpler tool.

Note that the only two arguments supplied are the RTF file handle (as before) and the name of the PNG file written by Stata previously. A simple design philosophy was adopted as it is possible to produce the final formatted version in the word processor to the user's preference. 
This utility has a sole option, leftoffset, which specifies (in $\mathrm{mm}$ ) the distance of the left-hand of the image from the left-hand page margin. Negative values are allowed to position it to the left of this margin.

This utility builds upon rtfutil's rtflink program, except that rtflink inserts a link to an image, and insertimagert $f$ inserts a copy of the actual image.

Full details are given in the insertimagert $f$ help file. If the required image file is missing, this function will gracefully fail, without corrupting the output RTF document.

\section{Example: putting it all together}

Here is a complete example which brings together the thoughts in the previous sections.

The document shown below is produced by the Stata code following it.

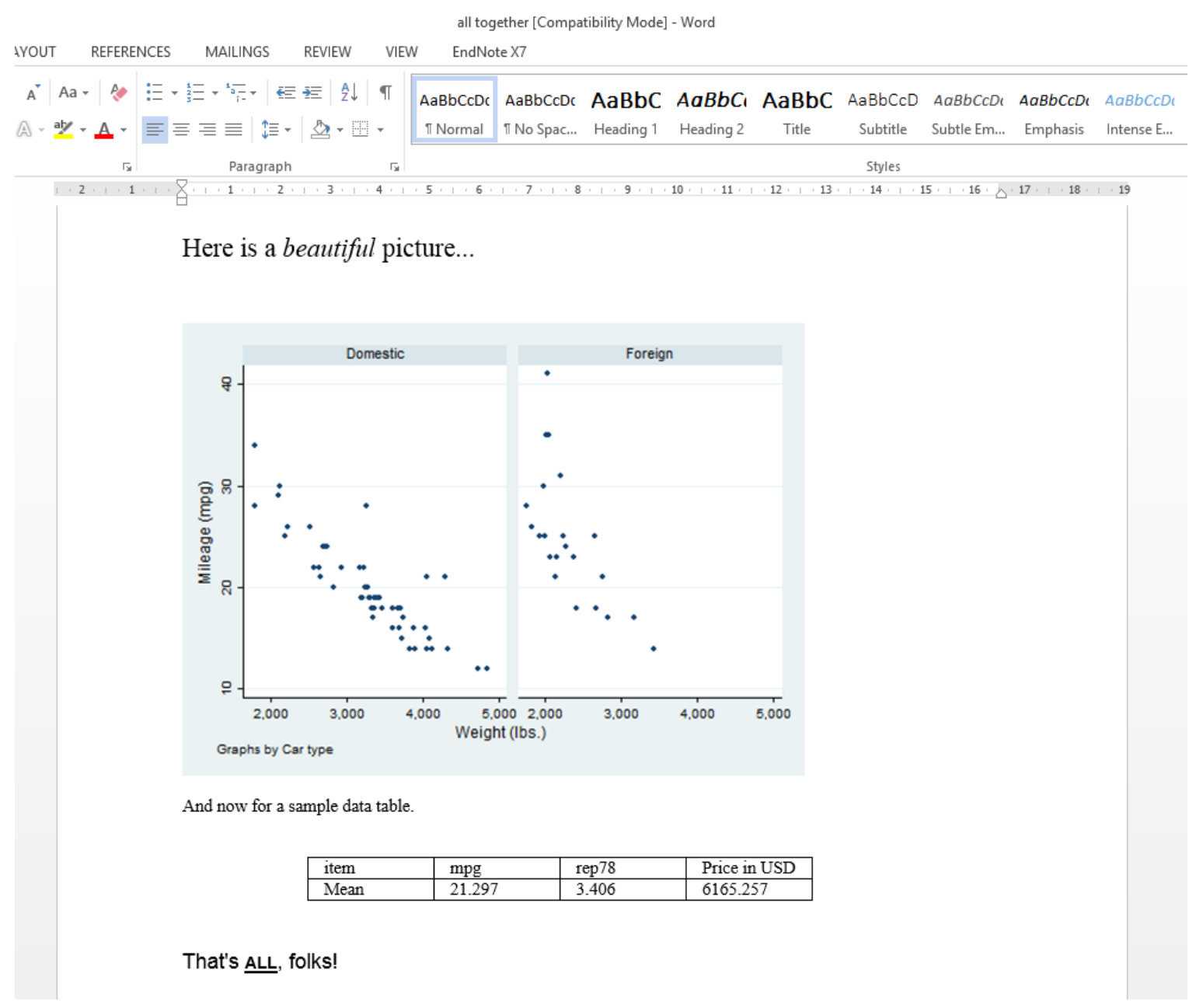

Notice the table set up by referencing the auto mean results in the following code. 
// This is file alltogether.do

clear all

sysuse auto, clear

set graphics on

scatter mpg weight, by (foreign)

global pngfile = "myplot.png"

graph export "\$pngfile", replace // Save graphics file to disk.

// set up output file.

global outputRTF = "all together.rtf"; // The name of the new RTF file

file open handlel using "\$outputRTF", write replace

initiatertf handle1

file write handle1 " $\backslash$ pard" // Start new paragraph

file write handlel "\fl\fs30 Here is a $\{\backslash i$ beautiful\} picture... \line \line"

file write handle1 "\par\}" // Close paragraph

insertimagertf handle1 "\$pngfile", leftoffset(0) // Adds graph into document. rm "\$pngfile" // Delete file (if desired).

$1 / 1 / 1 / 1 / 1 / 1 / 1 / 1 / 1 / 1 / 1 / 1 / 1 / 1 / 1 / 1 / 1 / 1 / 1 / 1 / 1 / 1 / 1 /$

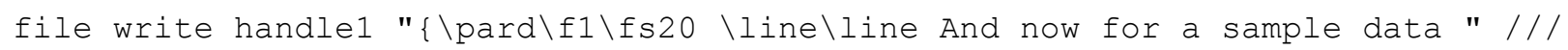

"table. \line \line \par\}"

file write handle1 "\{" n / / Start of table

tabstat mpg rep78 price, save

// This is a semi-automated way of obtaining the values desired:

mat mydata $=r($ StatTotal $)$

local cols = colsof (mydata) // Number of columns

forvalues colnum $=1 /$ 'cols' $^{\prime}$;

global d'colnum' : display $010.3 \mathrm{f}$ mydata[1, 'colnum']

\}

// Or you can do it manually, like this (no formatting here):

//global d1 = mydata $[1,1]$

$/ /$ global d2 = mydata $[1,2]$

/ /global d3 = mydata $[1,3]$

addrowrtf handle1 item mpg rep78 "Price in USD"

addrowrtf handle1 Mean \$d1 $\$ d 2 \quad \$ d 3$

file write handlel "\}" _n // End of table

$1 / 1 / 1 / 1 / 1 / 1 / 1 / 1 / 1 / 1 / 1 / 1 / 1 / 1 / 1 / 1 / 1 / 1 / 1 / 1 / 1 /$

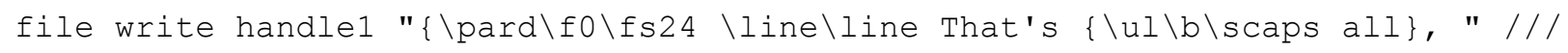

"folks! \line \line \par\}"

endrtf handle1

file close handlel

clear all 


\section{Known issues}

As stated previously, both utilities have deliberately been designed to be as easy to use as possible, given the complexities of RTF usage. Although there are a number of options to control output, the final RTF document will be viewed in a word processor and can be edited to the user's taste therein.

With addrowrtf, a truly long list of arguments (cell values) for a single row may well cause the table row to extend past the right-hand edge of the page in some cases. It may be possible to keep such a long table row within the page margins by judicious use of options.

\section{Conclusion}

Using Stata to produce RTF files can be tricky. Two utilities have been introduced; it is hoped that these will aid users who wish to automatically generate RTF documents from Stata.

\section{Acknowledgements}

This work was supported by Cancer Research UK C8162/A16892. I wish to thank Stata Technical Support, especially Derek Wagner who assisted with the conversion from byte values to hexadecimal values in the insertimagertf utility. I also thank Dr. Francesca Pesola and Professor Peter Sasieni, both of Queen Mary University of London, for their helpful comments and suggestions. The suggestions of an anonymous reviewer greatly improved the final submission.

\section{References}

Burke, Sean M. 2003. RTF Pocket Guide. Sebastopol: O'Reilly \& Associates.

Microsoft Corporation. 2008. "Word 2007: Rich Text Format (RTF) Specification, version 1.9.1." 20th March. Accessed October 18th, 2016. https://www.microsoft.com/en$\mathrm{gb} /$ download/details.aspx?id=10725.

\section{About the author}

Matthew Gillman is a statistical programmer at Queen Mary University of London. 